\title{
RELIABILITY OF ANTI-MYCOBACTERIAL DRUG SUSCEPTIBILITY TESTING AND IMPORTANCE OF ACCREDITATION OF LABORATORY PERFORMING THE TEST
}

\author{
Sharma KK, Jain NK, Jindal S, Jain N \\ Hospital for Chest Diseases and Tuberculosis, SMS Medical College, Jaipur 302 016, Rajastan, India
}

\begin{abstract}
Introduction: Tuberculosis (TB) is one of the major causes of disease and death in the developing world. World Health Organization recommends that drug resistance surveillance should be carried out regularly in high-burden countries, not only to determine the level of drug resistant TB, but also to strengthen the laboratory capacity. The objective of the study was to evaluate drug susceptibility test (DST) and to observe on DST reports with high variability between the results of two laboratories.
\end{abstract}

Methodology: The study was conducted at Hospital for Chest Diseases and Tuberculosis, SMS Medical College, Jaipur. This was an observational prospective study to analyze DST results, obtained from two different laboratories (Lab A and Lab B), of newly diagnosed sputum positive pulmonary tuberculosis patients registered on DOTS category I. Treatment outcome of patients was obtained from records at the referred clinics and hospitals.

Results: Higher proportion of resistance was observed from Lab A (29\%) than Lab B (16\%), Proportion of MDR strains were higher from Lab $A(12.5 \%)$ than Lab B $(6.0 \%)$. Out of the patients whose treatment outcome were available, proportion of patients with treatment success were almost similar in both cohorts $(84.6 \%$ vs. $85.2 \%)$, despite the difference in DST results. Success rate after treatment from Lab A was $60 \%$ even in patients with MDR strain, compared to $22 \%$ from Lab B.

Conclusions: Disparity in DST results was observed from the two laboratories (non accredited and accredited) which does not correlate with the treatment outcome. This study points towards the need of proper quality assurance of the drug sensitivity testing in Mycobacterial testing laboratories.

Key words: Tuberculosis, Drug susceptibility, Accreditation, Laboratory

\section{INTRODUCTION}

Tuberculosis (TB) is one of the major causes of disease and death in the developing world. Though modern drug treatment is effective in many cases, emergence of drug resistance especially multidrug resistant (MDR) TB has become a significant public health problem in a number of countries and

\section{Correspondence:}

Dr. Krishna K. Sharma

M.B., B.S., M.D.

Specialist (Pulmonary Medicine), ESIC Hospital, Bhiwadi

EM-II/101, BDI Sunshine City, Bhiwadi,

Rajasthan 301 019, India

E-mail: kksharma@yahoo.com a hindrance to effective TB control. ${ }^{1}$ In high-burden countries, World Health Organization (WHO) recommends that drug resistance surveillance should be done regularly, not only to determine the level of drug Resistant TB, but also to strengthen the laboratory capacity. ${ }^{2}$

Diagnosis and control of tuberculosis is difficult to achieve as it involves composite measures which may lead to drug related side effects, disruption of daily life, and social isolation. So, it is very important that the methods used to diagnose tuberculosis should be extremely precise. ${ }^{3}$ Albeit the development of many newer diagnostic methods, timely and proper use of TB culture may have an impact on TB rates in high-burden countries. Studies have shown that cultures 
of Mycobacterium tuberculosis from clinical specimens is presently close to the "gold standard" for diagnosis of TB. ${ }^{4-7}$ However, sometimes it can be misguiding especially due to the presence of interlab variability; it was also interesting to observe that assessment of resistance was different among laboratories, even when the same methods are applied. ${ }^{8,9}$

An erroneous or reprehensible drug sensitivity testing (DST) result may lead to recognition of susceptible cases as resistant or vice versa and may lead to inappropriate or inadequate treatment which may affect the treatment outcome. ${ }^{8}$ So, in high burden resource-poor countries, it is essential to carefully prioritize the tools of diagnosis with clinical relevance for maximal utilization of available healthcare facilities. This prospective analysis was conducted to evaluate drug resistance among newly diagnosed sputum positive cases of tuberculosis and to observe if there is any effect of variable DST on treatment outcome of these patients.

\section{METHODOLOGY}

\section{Setting}

The present study was conducted at the Hospital for Chest Diseases and Tuberculosis, SMS Medical College, Jaipur, a large academic hospital serving the population of the western part of India. In the hospital, the diagnosis of pulmonary TB is based on sputum smear microscopy following the National TB Programme recommendations. ${ }^{10}$ There is a TB laboratory register where recording of all patients' data along with test results is entered. All TB patients are registered at the hospital DOTS centre, and are referred to specific clinics or hospitals for treatment.

\section{Design}

This was an observational prospective study of newly diagnosed cases of tuberculosis attending outpatient and inpatient department of the hospital, from August 2008 to May 2010. A Structured standard questionnaire was used for the interview, subjects having positive sputum smear were included in the study. Patient with anti-TB treatment history of more than one month of treatment and patients with co-morbidities (diabetes, renal failure, hepatitis, HIV infection and other immunocompromised diseases) were excluded. Patients were randomized by simple random sampling for sending their sputum specimens to either of the two laboratories performing routine mycobacterial culture and DST, for evaluation to know the initial drug resistance (IDR). After sending sputum samples, all patients included in the study were registered in revised national tuberculosis control programme (RNTCP) and put on treatment under DOTS Category I.

\section{Laboratory Methods}

Pre-treatment sputum samples were sent randomly to the two laboratories. Although it was originally planned to culture two sputum samples per patient, due to logistic and cost constrains only one pretreatment sputum specimen could be collected from each patient and transported to laboratories. Cultures were done on LowensteinJensen (L-J) medium by modified Petroff's method. Cultures were incubated at $37^{\circ} \mathrm{C}$ and read for growth weekly for eight weeks. Isolates were identified as mycobacteria by smear microscopy and as M. tuberculosis by their slow growth rate, colony morphology, inability to grow on L-J media containing $p$-nitrobenzoic acid (PNB), niacin test and catalase test. ${ }^{11}$ DST was carried out by the economic variant of 1 per cent proportion method for all drugs except pyrazinamide which was tested by the resistance-ratio method. The tested drugs and their critical concentrations (in $\mu \mathrm{g} /$ $\mathrm{ml}$ ) were as follows: isoniazid $(\mathrm{H})-0.2$, rifampicin (R)-40, pyrazinamide (Z)-100, ethambutol (E)-2 and streptomycin (S)-4. ${ }^{9}$ The laboratory methods were uniform as per the standard operating procedure manual, on all samples and in both the laboratories. ${ }^{11,12}$

On availability of DST reports, high variability was observed between the results of two laboratories. Thus, two cohorts were formed on the basis of DST from two laboratories, first cohort (Lab A) with DST from non-accredited laboratory performing routine mycobacterial culture and sensitivity tests and second cohort (Lab B) with DST from accredited intermediate reference laboratory (IRL), where external quality control procedures were in place with monitoring by WHO Supra National Reference Laboratory. 
So, Second phase of study was planned, which is the basis of this article; to observe if there is any effect of variable DST on treatment outcome of the patients. Patient from both cohorts were followed at the completion of treatment, and treatment outcome was recorded from treatment register at clinics/ hospitals providing DOTS treatment.

\section{Data collection}

Hospital and laboratory data were collected by researchers. Names of culture-positive patients were checked against the registrations. Clinic visits were done by two field workers. At least two attempts were made to locate each patient at these follow-up visits made between October 2009 and May 2010.

\section{Case definitions}

Standard international definitions were used to define the treatment outcomes. ${ }^{13}$

Data analysis

The data collected were analyzed by using Microsoft Excel and SPSS (v10.0) computer software. Chisquare and "student- $t$ " test and proportion tests were applied for statistical significance. A $P$ value $\leq 0.05$ was considered statistically significant.

\section{Ethics}

Permission was obtained from relevant managers and senior clinicians and the study was approved by the Institutional Review Board of SMS medical college, Jaipur, India.

\section{RESULTS}

Variability in DST results

Laboratory results were available for 195 specimens from Lab A and 227 specimens from Lab B. A significantly higher proportion of resistance was observed from Lab A than Lab B $(29 \%$ vs $16 \%, x^{2}=9.3, p=0.002$ ) (Table 1). Out of total specimens, cultures were positive in $143(73.3 \%)$ of Lab A and $183(80.5 \%)$ of Lab B. Proportion of MDR strains were higher from Lab A (12.5\%) than Lab B (6.0\%). Lab A reported near to three times rifampicin resistance compared to $\mathrm{Lab} B(16.7 \%$ vs $\left.6.0 \%, X^{2}=8.6, p=0.003\right)$. A high proportion (4.1\%) of non-MDR rifampicin resistance was detected from Lab A (Table 2).

\begin{tabular}{|c|c|c|}
\hline & Lab A, n (\%) & Lab B, n (\%) \\
\hline Specimens tested & $195(100)$ & $227(100)$ \\
\hline $\begin{array}{l}\text { Negative culture/ } \\
\text { contaminated }\end{array}$ & $52(26.6)$ & 44 (19.3) \\
\hline Positive culture & $143(73.3)$ & $183(80.5)$ \\
\hline Susceptible to all & $86(44.1)$ & $146(64.3)$ \\
\hline $\begin{array}{l}\text { Resistant to at least one } \\
\text { drug }^{\#}\end{array}$ & $57(29.2)$ & $37(16.2)$ \\
\hline
\end{tabular}

\#Resistance to the drug in question, either alone or in combination with resistance to others.

Table 2. Number of culture-positive patients and drug sensitivity pattern from two laboratories

\begin{tabular}{|l|l|l|}
\hline & $\begin{array}{c}\text { Lab A (n=143), } \\
\mathbf{n}(\%)\end{array}$ & $\begin{array}{c}\text { Lab B (n=183), } \\
\mathbf{n}(\%)\end{array}$ \\
\hline Susceptible to all & $86(60.2)$ & $146(79.7)$ \\
\hline $\begin{array}{l}\text { Resistant to at } \\
\text { least one drug }\end{array}$ & $57(39.8)$ & $37(20.2)$ \\
\hline $\mathrm{H}$ & $39(27.2)$ & $34(18.5)$ \\
\hline $\mathrm{S}$ & $29(20.2)$ & $18(9.8)$ \\
\hline $\mathrm{R}$ & $24(16.7)$ & $11(6.0)$ \\
\hline $\mathrm{E}$ & $22(15.3)$ & $14(7.6)$ \\
\hline Z & $4(2.8)$ & $2(1.1)$ \\
\hline $\begin{array}{l}\text { Multi Drug } \\
\text { Resistance }\end{array}$ & $18(12.5)$ & $11(6.0)$ \\
\hline
\end{tabular}

Treatment outcome of culture positive patients

Of the 143 patients with culture-positive results from Lab A and 183 from Lab B, treatment outcomes of 124 and 169 patients respectively from each cohort were available, rest of the patients either did not reported to the referral clinics or were migrated/ transferred out.

Treatment outcome and effect of Variability in DST results

Proportion of patients with treatment success (cure/ treatment completed) after completion of DOTS were almost similar in both cohorts $(84.6 \%$ vs. $85.2 \%$ ), despite of variability in DST results (Table 3, 4). Also it was almost similar in patients with susceptible strains from both cohorts $(89.4 \%$ and $91.4 \%$ ). A higher proportion of success was seen in patients with resistant strains other than MDR from Lab A (86\%) than Lab B (78\%). More than half of the subjects with MDR strain from 
Lab A (60\%) got success with DOTS category I, whereas poor success rate was noted among same set of patients from second cohort (22\%). Less proportion of patients having MDR strains had adverse outcome (treatment failure/ death) from Lab A (13\%) than Lab B (55\%), further questioning about DST results from Lab $A$.

\begin{tabular}{|l|l|l|l|l|}
\hline \multicolumn{5}{|l|}{$\begin{array}{l}\text { Table 3.Treatment Outcome of Patients with DST from } \\
\text { Lab A }\end{array}$} \\
\hline & \multicolumn{4}{|c|}{ TREATMENT OUTCOME } \\
\cline { 2 - 5 } & Success & Default & Failure & Death \\
\hline $\begin{array}{l}\text { Sensitive to } \\
\text { all }(\mathrm{n}=66)\end{array}$ & $59(89.4)$ & $5(7.5)$ & $2(3.0)$ & - \\
\hline $\begin{array}{l}\text { MDR } \\
(\mathrm{n}=15)\end{array}$ & $9(60)$ & $4(26.6)$ & $1(6.6)$ & $1(6.6)$ \\
\hline $\begin{array}{l}\text { Other } \\
\text { Resistance } \\
(\mathrm{n}=43)\end{array}$ & $37(86.0)$ & $2(4.6)$ & $3(7.0)$ & $1(2.3)$ \\
\hline $\begin{array}{l}\text { Total } \\
(\mathrm{n}=124)\end{array}$ & $105(84.6)$ & $11(8.8)$ & $6(4.8)$ & $2(1.6)$ \\
\hline
\end{tabular}

* Success $=$ Cured + treatment completed

\begin{tabular}{|c|c|c|c|c|}
\hline & \multicolumn{4}{|c|}{ TREATMENT OUTCOME } \\
\hline & Success* & Default & Failure & Death \\
\hline $\begin{array}{l}\text { Sensitive to } \\
\text { all }(n=128)\end{array}$ & $117(91.4)$ & $8(6.2)$ & $3(2.3)$ & $5^{-}$ \\
\hline $\begin{array}{l}\text { MDR } \\
(n=9)\end{array}$ & $2(22.2)$ & $2(22.2)$ & $3(33.3)$ & $2(22.2)$ \\
\hline $\begin{array}{l}\text { Other } \\
\text { Resistance } \\
(n=32)\end{array}$ & $25(78.1)$ & $3(9.3)$ & $3(9.3)$ & $1(3.1)$ \\
\hline $\begin{array}{l}\text { Total } \\
(\mathrm{n}=169)\end{array}$ & 144 (85.2) & $13(7.7)$ & $9(5.3)$ & $3(1.7)$ \\
\hline
\end{tabular}

* Success $=$ Cured + treatment completed

\section{DISCUSSION}

Findings of the present study showed variation in DST results between the two labs, non accredited lab showing almost double drug resistance compared to accredited lab. On follow up for outcome of treatment, almost similar success and failure rate (Table 3,4$)$ were observed among patients with drug sensitive and drug resistance (other than MDR) TB, despite of variability in drug resistance results, suggesting that standard SCC regimen had been effective in a majority of patients. ${ }^{14}$ The concern was variable outcome in patients with MDR TB, as in the patients with MDR strain, the initial strain is already resistant to both isoniazid and rifampicin, and virtually there is no drug during maintenance phase of short course chemotherapy (SCC), which possibly results in treatment failure. ${ }^{15}$ But good success and less failure rate was noted in these patients with DST from Lab A in contrast to Lab B, which is unlikely in presence of true MDR TB, questioning the reliability of the DST results. Laboratory inaccuracy is also indicated by presence of high rifampicin resistance from Lab A in contrast to Lab $B$ and high (4.1\%) non-MDR rifampicin resistance from Lab A. Rifampicin resistance is usually accompanied by isoniazid resistance, presence of isolated rifampicin resistance is very uncommon and good marker for accuracy of laboratory; more than $3 \%$ reporting of non-MDR rifampicin resistance indicate errors in either rifampicin or isoniazid testing. ${ }^{16}$

Given the inter-lab variability of the test, poor reliability warrants careful interpretation, as overall response rate of MDR TB is far below that of drugsusceptible TB, if susceptible strains are reported as resistant, regimens may be changed unnecessarily and reserve drugs may be introduced which are more toxic, less potent and more costly than drugs used for primary treatment. ${ }^{3,17-19}$

The main factors which may be responsible to influence DST results include inappropriate standardization of inoculum preparation, impure culture media and improper test environment. ${ }^{9}$ Other factors which may be accountable include contamination of clinical devices, clerical errors, laboratory cross contamination, staff shortages relative to the workloads, lack of experience, and most important, failure of the clinician to discuss incongruent results with laboratory staff. 3020 Accuracy is even more difficult to achieve in countries were skilled manpower and adequate facilities for such tests are scarce. ${ }^{21}$

Present study highlights the fact that improper susceptibility testing for $M$. tuberculosis is not rare and discrepancies are seen between in vitro and in vivo results and accreditation of laboratories with good quality control should be done to minimize these discrepancies. All laboratories should carry out a thorough internal quality control programme. Analyses and diagnostic services should be accredited with meticulous participation in relevant proficiency schemes to follow strict guidelines and 
recommendations. Similarly, where a licensing system exists, laboratories should be licensed to perform TB-related microbiological activity ${ }^{22}$. Standardization of Laboratories is one important step which may help in strict quality control with the purpose of optimizing the clinical relevance of DST results.

These issues call for physicians' attention when using the results from drug-susceptibility testing for case management. The earlier studies providing clinical follow-up of patients with false positive cultures demonstrates a lack of awareness among clinicians and laboratory personnel of the possibility of false-positive cultures and showed that, patients having false-positive cultures were treated for tuberculosis, some of whom experienced toxicity from multidrug tuberculosis treatment. ${ }^{23-25}$ First, clinicians should evaluate results of DST critically; and ensure that it should be compatible with patients' clinical response. Second, it should be ensured that DST to be done from standard accredited laboratories, whenever possible and if facilities are not available patient should be referred to higher center. Third and most importantly, patients' clinical response along with sputum conversion should be given an upper consideration before switching on to the regimens based on drug susceptibility.

Some limitations of the study may be taken into account. The main limitation is that the results from only two Laboratories are compared; one of which is accredited and other non-accredited, results would have been more reproducible if more number of laboratories with large sample size were compared. But by comparing the non accredited lab to accredited, we have reinforced the need for accreditation of lab for DST. Further, the possibility of misclassification of retreatment cases as new cases cannot be ruled out, even though proper history was taken and previous treatment records were documented if available. We acknowledge that due to the nature of study design, each laboratory reported on different set of patients and specimens, as randomization of specimen to one or the other laboratory was done; bias could have been introduced if covariates, such as treatment history, age or severity of disease, were associated with certain group of patients. However, proper randomization was carried out using the same testing conditions for each subject and on comparison between the two groups for general characteristics; no statistically significant difference was observed between the groups. We also acknowledge that to get the exact scenario of DST variability among two laboratories; same samples must be processed by both the laboratories for drug resistance.

Keeping in view the implications of DST in proper diagnosis of MDR TB for effective tuberculosis control, we suggest standardization and quality control of mycobacterial laboratory methods with careful clinical correlation by clinicians for effectual management of MDR TB, curbing the development of drug resistance.

\section{ACKNOWLEDGEMENTS}

The authors express their gratitude to thank Prof. S. P. Agnihotri, MD and Dr. Mahesh Gupta, MD for reviewing the manuscript and constructive suggestions at various stages of the study. We sincerely thank staff of this hospital and also acknowledge the efforts of the health visitors in obtaining the relevant data efficiently and in a short period. This work would not have been successfully completed without the cooperation of all study patients and their family members.

\section{REFERENCES}

1. RNTCP Status Report-TB INDIA 2010. The Central TB Division 2010. India.

2. World Health Organization. Guidelines for surveillance of drug resistance in tuberculosis. WHO/HTM/TB/2009.422. Geneva, Switzerland: WHO, 2009.

3. Burman WJ, Reves RR. Review of false positive cultures for mycobacterium tuberculosis and recommendations for avoiding unnecessary treatment. Clinical Infectious Diseases 2000;3:1390-95.

4. Moore DA, Evans CA, Gilman RH, Caviedes L, Coronel J, Vivar A, et al. Microscopic-observation drug-susceptibility assay for the diagnosis of TB. N Engl J Med 2006;355:1539-50.

5. Perkins MD, Cunningham J. Facing the crisis: improving the diagnosis of tuberculosis in the HIV era. J Infect Dis 2007;196:S15-S27. 
6. Dowdy DW, Chaisson RE, Maartens EL, Dorman SE. Impact of enhanced tuberculosis diagnosis in South Africa: a mathematical model of expanded culture and drug susceptibility testing. Proc Natl Acad Sci U S A 2008;105:11293-98.

7. Getahun $H$, Harrington M, O'Brien R, Nunn P. Diagnosis of smear-negative pulmonary tuberculosis in people with HIV infection or AIDS in resource-constrained settings: informing urgent policy changes. Lancet 2007;369:2042-49.

8. Kim SJ. Drug-susceptibility testing in tuberculosis: methods and reliability of results. Eur Respir $\mathrm{J}$ 2005;25:564-69.

9. WHO/IUATLD Global project on anti-tuberculosis drug resistance surveillance. Guidelines for surveillance of drug resistance in tuberculosis. WHO/TB/96.216. Geneva, Switzerland: WHO, 1996.

10. Guidelines for Quality Assurance of smear microscopy for diagnosing tuberculosis. The Central TB Division 2005. India.

11. Manual on Isolation, Identification and Sensitivity testing of Mycobacterium tuberculosis, $2^{\text {nd }}$ Edition. National Tuberculosis Institute 1998. India.

12. International Union against Tuberculosis and Lung Disease (IUATLD). The Public Health Service National Tuberculosis Reference Laboratory and the National Laboratory Network - Minimum Requirements, Role and Operation in a LowIncome Country. Paris: IUATLD, 1998;72-76.

13. Revised National Tuberculosis Control Program (RNTCP), Technical guidelines for TB control. The Central TB Division 1997; India.

14. Espinal MA, Kim SJ, Suarez PG, Kam KM, Khomenko AG, Migliori GB, et al. Drug-Resistant Tuberculosis: Treatment Outcomes in 6 Countries. JAMA 2000;283:2537-45.

15. Costello HD, Caras GJ, Snider DE Jr. Drug Resistance Among Previously Treated Tuberculosis Patients-A Brief Report. Am. Rev. Respi.Dis 1980;121:313-16.
16. WHO/IUATLD Global project on anti-tuberculosis drug resistance surveillance. Anti-tuberculosis Drug Resistance in the World, Report No. 4. WHO/ HTM/TB/2008.394. Geneva, Switzerland: WHO, 2008.

17. Katiyar SK, Prakash S, Bihari S. Anti-mycobacterial susceptibility as a tool in the management of multidrug resistant tuberculosis in resource poor countries - How reliable is it?. Indian Journal for the Practising Doctor 2006;3:11-12.

18. Fox W. General considerations on the choice and control of chemotherapy in pulmonary tuberculosis. Bulletin of the International Union Against Tuberculosis 1972;47:51-71.

19. Iseman MD. Treatment of multidrug-resistant tuberculosis. N Engl J Med 1993;329:784-91.

20. Nitta A, Davidson PT, de Koning ML, Kilman J. Misdiagnosis of multi-drug resistant tuberculosis possibly due to laboratory errors. JAMA 1996;276:1980-3.

21. Espinal M. How reliable are drug susceptibility tests?. In: Frieden T, editor. Toman's Tuberculosis - Case Detection, Treatment, and Monitoring. 2nd ed. Geneva, Switzerland: WHO; 2004; 211.

22. World Health Organisation, Geneva. Guidelines for the programmatic management of drug-resistant tuberculosis. WHO/HTM/tb/2006.361.

23. Burman WJ, Stone BL, Reves RR, Wilson M L, Yang Z, El-Hajj $\mathrm{H}$ et al. The incidence of falsepositive cultures of Mycobacterium tuberculosis. Am J Respir Crit Care Med 1997;55:321-6.

24. Cronin W, Rodriguez E, Valway S, Bur S, Hooper $\mathrm{N}$, Smithwick R, et al. Pseudo-outbreak of tuberculosis in an acute-care general hospital: epidemiology and clinical implications. Infect Control Hosp Epidemiol 1998;19:345-7.

25. Centers for Disease Control. Multiple misdiagnoses of tuberculosis resulting from laboratory errorWisconsin. MMWR Morb Mortal Wkly Rep 1997;46:797-801. 